\title{
Gate Splitting in LOTOS Specifications Using Abstract Interpretation *
}

\author{
Fosca Giannotti and Diego Latella \\ CNR, Ist. CNUCE, via S.Maria 36, I56126 Pisa Italy \\ email: fosca@cnuce.cnr.it latella@fdt.cnuce.cnr.it **
}

\begin{abstract}
In this paper ${ }^{1}$ a technique for an efficient solution to the problem of gate splitting in LOTOS specifications is presented. The transformation problem is part of a design methodology based on the specification language LOTOS. The problem is formally defined. The technique is based on Abstract Interpretation which is used for approximating the sets of possible values which LOTOS value expressions can evaluate to. The originality of the proposed approach stems from the fact that the abstract domain as well as abstract functions are generated automatically from the LOTOS specification to be transformed. The abstract interpretation as well as the transformation are proved correct.
\end{abstract}

\section{Introduction}

A software development methodology based on the ISO standard specification language $\operatorname{LOTOS}[2,3,20]$ and on the notion of correctness preserving transformations [18] has been developed as a major result of the ESPRIT-LOTOSPHERE Project $[14,15,16,17]$.

Gate splitting is a simple, yet useful, transformation which, given a specification $P$, hereafter called the input specification, returns a specification $Q$ insisting on a set of gates different from that of the input specification but preserving the information on the behaviour of $P$. In other words the transition systems of $P$ and $Q$ are isomorphic; they share the same non-deterministic structure and the actions of $Q$ are obtained via an injective renaming of those of $P$. The gates of $Q$ are obtained from those of $\mathrm{P}$ by means of splitting: a certain gate $g$ in $P$ can be split into different gates $g_{1}, g_{2}, \ldots, g_{k}$ in $Q$. For each action occurring in each $g_{i}$ in $Q$, there will be a uniquely determined action of $P$ occurring in $g$, which can be recognized by interaction parameters and vice-versa. Thus, no information on $P$ is lost in $Q$.

There are different motivations for this transformation at different steps of the development trajectory from abstract specification to concrete realization [14] including the need of splitting an abstract interaction point into a set of communication

* This work has been done within the ESPRIT Project Ref. 2304 - LOTOSPHERE and has been partially funded by the CNR-NATO Advanced Fellowships Program.

** Current address of Diego Latella: Univ. of Twente, Dept. of Comp. Science, PO BOX 217, 7500 AE Enschede, The Netherlands email: latella@cs.utwente.nl

1 An extended abstract of the present paper has already been published in the Proceedings of the "Workshop on Static Analysis" LaBRI - Univ. of Bordeaux I 23-25 Sept. 1992. 
channels when the specification is matched against an implementation architecture or when the implementation language imposes constraints on communication channels (e.g. forcing them to be typed). Finally, splitting a gate can facilitate increasing potential parallelism, to be obtained by means of other transformations [22, 13, 18].

In the present paper we show how Abstract Interpretation (AI) can be used in order to get an efficient solution to the gate splitting problem, when the input specification satisfies some syntactical constraints. Such constraints give the abstract data type (ADT) part of the specification a functional programming language style.

AI of programs is a static analysis technique aimed at gathering information about the dynamic behaviour of a program or specification, to be used by compilers, partial evaluators and debuggers, or merely as documentation $[1,4,5,6,7]$. AI consists in assigning language constructs non-standard (i.e. abstract) meaning which approximate standard semantics.

The original contribution of this paper stems from the fact that the abstract domain for abstract interpretation as well as abstract functions are automatically generated from the input specification and heavely depend on it. With respect to this, speaking about Abstract Compilation, more than AI would be more appropriate. This is quite uncommon in traditional use of AI where the definition of abstract domains and functions is left to the user of the technique who is usually forced to rely on "ad hoc" solutions.

In this paper it is assumed the reader is familiar both with LOTOS and with AI. We believe that the key ideas for the above mentioned automatic construction of the abstract domain are largely independent from LOTOS and therefore can be applied to different languages. Nevertheless, we shall make explicit reference to LOTOS here in order to show them working on a real specification language.

In Sect.2 a possible formalization of the gate splitting problem is given and a solution is informally introduced. A formal solution to the problem is discussed in Sect.3. The solution is proved correct. The details of the proofs are omitted here, the interested reader being referred to [9]. Finally, in Sect.4 several improvements to our solution are briefly discussed.

\section{The problem of Gate Splitting}

In this paper we shall assume that a gate can be split only according to the values exchanged throughout it. Thus the Gate Rearrangement Criterion (GRC) will be specified as a function $\mathcal{R}$ : Gates $\rightarrow$ Values $\rightarrow$ Gates where Gates and Values, denote respectively the set of LOTOS gate identifiers and data values. Moreover, in order for the transformation to make sense, we shall require that range $(\mathcal{R})$ be finite.

Consider for example the following LOTOS process definition ${ }^{2}$ :

\footnotetext{
${ }^{2}$ In the sequel no distinction will be made between process definitions and specifications. Also, the syntax of the ADT part is slightly different from that of standard LOTOS. The details on such a syntax will be given in Sect.3.1. Moreover, currying will be often used for functions [8] in the meta-notation.
} 


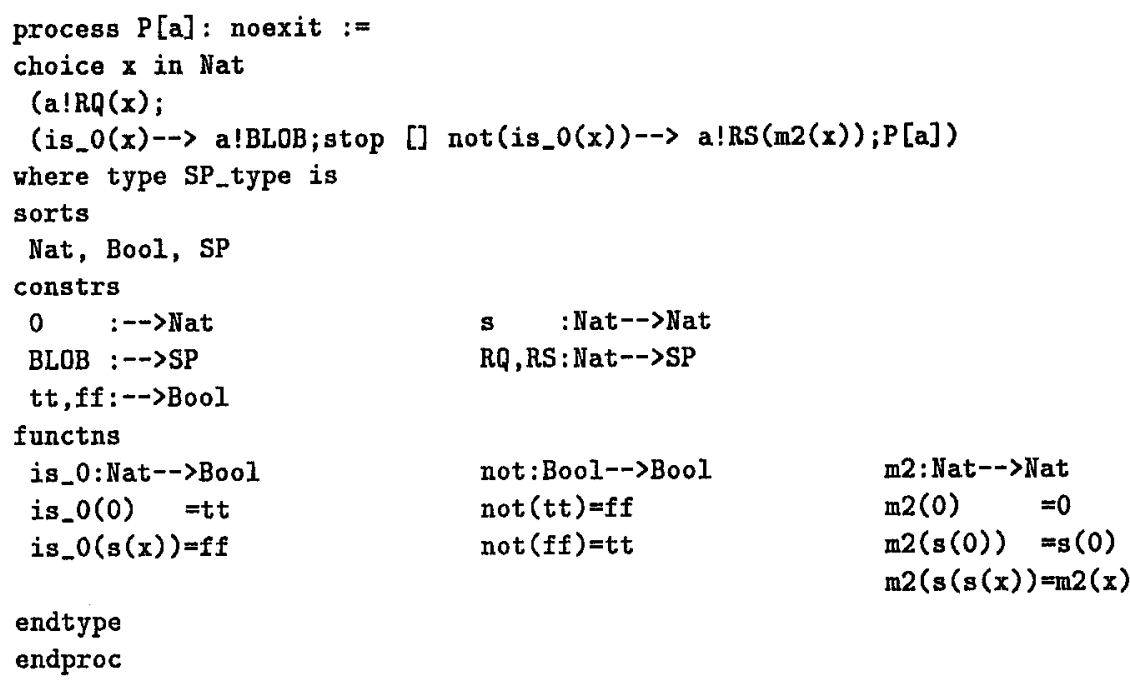

Process $P$ computes a function $m 2$ on any positive natural number $n$ received as a request $R Q(n)$ on gate $a$ and returns the result $R S(m 2(n))$ on the same gate. If the input value is 0 the process returns the constant $B L O B$ and stops. Suppose now we want to split gate $a$ in such a way that all the request actions will occur at either gate $r 0$ or $r$, according to the fact the input value is zero or a positive number. Moreover we want the $B L O B$ action occur on the customized gate $b$. Finally, if the result of the computation is 0 then the response action must accur on gate $s 0$, otherwise it will occur on gate $s$. Such informal criterion corresponds to the following GRC $\mathcal{G}$, which is defined only on gate $a$ :

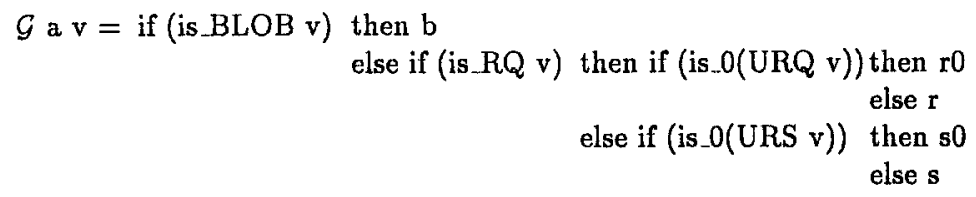

The above definition uses some functions defined as follows:

\begin{tabular}{|c|c|c|c|}
\hline URQ, URS : SP-->Nat & is_BLOB : $S P \rightarrow B$ BOOl & is_RQ: $\mathrm{SP}-->\mathrm{BOOl}$ & S: SP- ->Bool \\
\hline 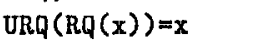 & $B(B L O B)=t t$ & is_RQ $(R Q(x))=t t$ & $S(x))=t$ \\
\hline URS (RS $(x$ & $\begin{array}{l}\text { is_BLOB }(R Q(x))=f f \\
\text { is_BLOB }(R S(x))=f f\end{array}$ & $\begin{array}{l}\text { is_RQ(BLOB) }=f f \\
\text { is_RQ }(R S(x))=f f\end{array}$ & $\begin{array}{l}\text { is_RS }(B L O B)=f f \\
\text { is_RS }(R Q(x))=f f\end{array}$ \\
\hline
\end{tabular}

So, the first step of our methodology consists in defining the GRC in terms of functions defined in the ADT part of the specification, possibly extended with some auxiliary functions ${ }^{3}$.

In order to formalize the transformation correctness requirement we need the following definitions, which extend the notion of strong bisimulation equivalence [11].

\footnotetext{
${ }^{3}$ Notice that the GRC itself is not part of the ADT specification because, in the general case it might contain tests on the type of data values, which cannot be defined within the LOTOS specification itself.
} 
Definition 1 Labeled Transition System. A Labeled Transition System is a 4tuple $\langle S, A, T, s\rangle$, where $S$ is a set of states, $A$ is a set of action labels, $T$ is a set of transition relations: given $a \in A$ the transition relation $\stackrel{a}{\rightarrow}$ is a binary relation over $S$. We shall often write $s_{1} \stackrel{a}{\rightarrow} s_{2}$ for $\left\langle s_{1}, s_{2}\right\rangle \in \stackrel{a}{\rightarrow}$. Finally $s \in S$ is the initial state.

Definition $2 \phi$-bisimulation relation. Let $S y s_{1}=\left\langle S_{1}, A_{1}, T_{1}, s 0_{1}>\right.$ and $S y s_{2}=$ $<S_{2}, A_{2}, T_{2}, s 0_{2}>$ be labeled transition systems. Let $\phi: A_{1} \longrightarrow A_{2}$ be a bijection. A $\phi$-bisimulation relation is any relation $R_{\phi} \subseteq S_{1} \times S_{2}$ such that:

$\forall a_{1} \in A_{1}, a_{2} \in A_{2}, s_{1} \in S_{1}, s_{2} \in S_{2}$ with $<s_{1}, s_{2}>\in R_{\phi}$

1. $s_{1} \stackrel{a_{1}}{\rightarrow} s_{1}^{\prime} \Rightarrow \exists s_{2}^{\prime}: s_{2} \stackrel{\phi a_{1}}{\rightarrow} s_{2}^{\prime}$ and $<s_{1}^{\prime}, s_{2}^{\prime}>\in R_{\phi}$

2. $s_{2} \stackrel{a_{2}}{\rightarrow} s_{2}^{\prime} \Rightarrow \exists s_{1}^{\prime}, a_{1}: s_{1} \stackrel{a_{1}}{\rightarrow} s_{1}^{\prime}$ and $a_{2}=\phi a_{1}$ and $<s_{1}^{\prime}, s_{2}^{\prime}>\in R_{\phi}$

Definition $3 \Phi$-bisimulation equivalence. $B_{1}$ and $B_{2}$ are $\Phi$-bisimulation equivalent, written $B_{1} \sim_{\Phi} B_{2}$, iff there exists a $\phi$-bisimulation $R_{\phi}$ such that $B_{1} R_{\phi} B_{2}$

Proposition 4. $\sim_{\Phi}$ is an equivalence relation.

Our transformation problem can then be formalized as follows, where for all process definitions $B$ Gates $(B)$ returns the set of gates which textually occur within $B$ and $L T S_{B}$ stands for the labeled transition system denoted by $B$ :

Given a process definition $P$ and a GRC $\mathcal{R}$ we want to get a process definition $Q$ such that $\operatorname{Gates}(Q)=\mathcal{R}(G a t e s(P) \times$ Values $)$ and $L T S_{P} \sim_{\Phi} L T S_{Q}$.

A possible solution for our sample process definition and GRC is the following one:

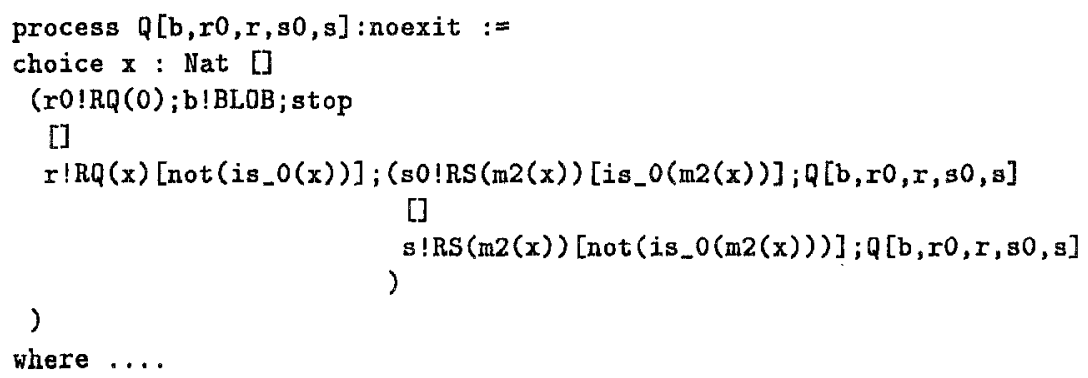

A close look at the above process $Q$ reveals that it is extremely useful for the transformation to have knowledge about the possible output values of the GRC when it is applied to values denoted by LOTOS value expressions. In fact, knowing that $\mathcal{G}$ a $B L O B=b$ we can translate $a ! B L O B ;$ stop into $b ! B L O B ;$ stop. On the other hand, since $m 2(x)$ may be any natural number and the set of possible values for $\mathcal{G}$ a $R S(n)$, for any natural number $n$ is $\{s 0, s\}$, we shall translate $a ! R S(m 2(x)) ; \ldots$ into a choice expression like the following one:

(so!RS $\left.(\mathrm{m} 2(x))\left[i s_{-} 0(\mathrm{~m} 2(x))\right] ; \ldots\right)$

[]

$\left(\operatorname{s!nS}(m 2(x))\left[\operatorname{not}\left(i s_{-} 0(m 2(x))\right)\right] ; \ldots\right)$ 
where the appropriate alternative will be selected by a selection predicate consistent with the definition of the GRC (for instance is $0(m 2(x))$ in the first alternative). Notice that complete lack of knowledge on the possible output values of $\mathcal{G}$ a $R S(n)$ would have resulted in a choice expression with five alternatives instead of two, one for each member of the codomain of $\mathcal{G}$, i.e. $\{b, r 0, r, s 0, s\}$.

Essentially the transformation acts on action prefix changing it into a choice expression where each alternative is an action prefix guarded by a selection predicate, the treatment of other constructs being mainly implied by these main changes. The number of alternatives of such choice expressions depend both on "how much" the GRC depends on actual data values and on the amount of knowledge on that which can be acquired from a static analysis of the input specification. In Sect. 3, we shall develop a technique for computing such a knowledge and for using it within the transformation.

\section{A Solution to the Problem of Gate Splitting}

In this section we shall describe in detail the solution we propose for the gate splitting problem. A major emphasis will be put on the static analysis technique we use for getting suitable knowledge on the GRC behaviour. The transformation itself is quite straightforward.

The key point is to get statically computable information on the possible values which the GRC as well as LOTOS value expressions can evaluate to in a given specification. AI can be used for finitely approximating the sets of those values. The level of uncertainty present in those approximations is determined by the amount of the information on both the "data flow" and the behaviour of functions defined in the ADT part of a specification which can be deduced by a static analysis of the specification.

In our example function $m 2$ is defined on $N a t$. The set $\left\{0, s(0), s\left(s\left(\perp_{N a t}\right)\right)\right\}$ can be chosen as a representation for $N a t$, where 0 and $s(0)$ are represented explicitly and $s\left(s\left(\perp_{N a t}\right)\right)$ represents $s(s(0)), s(s(s(0))), s(s(s(s(0)))) \ldots$ Notice that the above representation is finite (it contains only three elements) and complete (every term in $N a t$ is represented). Also, it is an abstraction of $N a t$ (the term $s\left(s\left(\perp_{N a t}\right)\right)$ intuitively stands for any natural number greater than 1 ). Moreover, it gives 0 and $s(0)$ a "special status" of distinguished elements; this is because those two terms are the only terms which occur explicitly in the text of the process definition.

Starting from $\left\{0, s(0), s\left(s\left(\perp_{N a t}\right)\right)\right\}$ we can use its power set as an abstraction for the set of sets on Nat. We must keep in mind that the elements of such an abstract domain, i.e. the abstract terms, are just approximations of sets on natural numbers in the sense that not all sets are explicitly represented. Some of them are only approximated by terms which represent sets in which they are included.

The abstract version $f^{\mathcal{A}}$ of a function $f$ is a function which gives information on the possible outcomes of $f$ when applied to sets of possible input values. $f^{\mathcal{A}}$ is defined on abstract terms and is then a finite function. Finally, the information on the possible values of value expressions is obtained via an abstract evaluation function $\mathcal{V}$, which maps LOTOS value expressions into abstract terms. 
In the following we shall describe the details of our technique for the automatic construction of the abstract domain and the procedure for getting the abstract functions out of the definition of their concrete counterpart.

\subsection{Abstract Framework}

In the sequel we shall assume that in the ADT part of the input specification constructor names will be kept separated from function names. Given the term $n\left(t_{1}, \ldots, t_{n}\right)$, we shall call it a constructor term if $n$ is constructor name and we shall call it a functor term if $n$ is a function name. Only functor terms may occur in the left-hand-side of equations. For the sake of notational simplicity, in this paper conditional equations are not dealt with and we also require that for each term $t$ of a given sort $s$ and function $f: s \rightarrow s^{\prime}$ there is exactly one equation in the definition of $f$ the left-hand-side of which matches with $t$ (this implies that the technique presented here does not deal with "error-driven" default values for functions as well as "overlapping patterns"). Let $S$ and $\Sigma$ respectively be the sorts set and the signature of the input specification. The word algebra over $\Sigma$ will be denoted by $T_{\Sigma} . T_{\Sigma}$ VVar denotes the algebra of terms with (sorted, free) variables. $T_{\Sigma_{c}}$ will denote the subset of $T_{\Sigma}$ containing only terms made up of constants and constructors applied to them recursively ${ }^{4} . A_{s}$ will denote the carrier of sort $s$ in the $\Sigma$-algebra $A$. We say that a sort $s$ is basic if whenever $K: s_{1}, \ldots, s_{n} \rightarrow s$ is a constructor of sort $s$, then $s_{1}=\ldots=s_{n}=s$. In this paper we shall consider only specifications in which the ADT part has at least one basic sort. The above notation will be used also for families of sets.

Abstract Domains The starting point for the construction of the abstract domains is the notion of partial term.

Definition 5 Partial term posets: $\left\langle\left(\left(\Pi_{\Sigma}\right)_{s}, \preceq_{s}\right)\right| s \in S>$. For all sorts $s$ the set of partial terms of sort $s$ is defined as the smallest set $\left(\Pi_{\Sigma}\right)_{s}$ such that:

1. $\perp_{s} \in\left(\Pi_{\Sigma}\right)_{s}$

2. for all constants $c: \rightarrow s, c \in\left(\Pi_{\Sigma}\right)_{s}$ and

3. for all constructors $k: s_{1}, \ldots, s_{n} \rightarrow s$ and partial terms $t_{i} \in\left(\Pi_{\Sigma}\right)_{s_{i}}$, $i=1 \ldots n, k\left(t_{1}, \ldots, t_{n}\right) \in\left(\Pi_{\Sigma}\right)_{s}$.

The partial order relation $\preceq_{s}$ is defined as the reflexive, anti-symmetric and transitive closure of relation $\sim_{s}$, which is the smallest set in $\left(I_{\Sigma}\right)_{s} \times\left(\Pi_{\Sigma}\right)_{s}$ such that:

1. $\perp_{s} \sim_{s} t$ for all $t \in\left(\Pi_{\Sigma}\right)_{s}$

2. $k\left(t_{1}, \ldots, t_{n}\right) \sim_{s} k\left(r_{1}, \ldots, r_{n}\right)$ iff $t_{i} \sim_{s_{i}} r_{i}$, for all $i=1, \ldots, n$.

As usual, $x \preceq_{8} y$ and $x \neq y$ will be denoted by $x \prec_{8} y$. Also, the subscript $s$ will be omitted when this will not give raise to ambiguities.

\footnotetext{
${ }^{4}$ Notice that due to the restriction of not having equations for constructors $T_{\Sigma_{c}}$ correctly represents $T_{\Sigma}$ modulo the equations for the functions in the ADT definition.
} 
Definition 6 Partial terms generators: $\pi_{s}$. For all sorts $s$ $\pi_{s}:\left(T_{\Sigma \cup V a r}\right)_{s} \rightarrow\left(\Pi_{\Sigma}\right)_{s}$ is defined as follows:

$\pi_{a}(c) \quad=c$ for all constants $c: \rightarrow s$

$\pi_{s}(x) \quad=\perp_{s}$ for all variables of sort $s$

$\pi_{s}\left(k\left(t_{1}, \ldots, t_{n}\right)\right)=k\left(\pi_{s_{1}}\left(t_{1}\right), \ldots, \pi_{s_{n}}\left(t_{n}\right)\right)$ for all constructor terms

$\pi_{s}\left(f\left(t_{1}, \ldots, t_{n}\right)\right)=\perp_{s} \quad$ for all functor terms

Intuitively, given a sort $s$, a partial term is a finite representation for a subset of $\left(T_{\Sigma}\right)_{s}$ and is obtained from a term in $\left(T_{\Sigma \cup V_{a r}}\right)_{s}$ where variables and functor terms are replaced by a special symbol, $\perp$, representing all values in $\left(T_{\Sigma}\right)_{\boldsymbol{s}^{\prime}}$ for some proper sort $s^{\prime}$. Any ground term $t$ simply represents the singleton $\{t\}$. For instance, in our example, 0 represents $\{0\}, \perp_{N \text { at }}$ represents $\{0, s(0), s(s(0)), s(s(s(0))), \ldots\}$, $s\left(\perp_{N a t}\right)$ represents $\{s(0), s(s(0)), s(s(s(0))), \ldots\}$ and so on. The intuitive meaning of the ordering relation is that $t_{1} \preceq t_{2}$ if $t_{1}$ is "less specified", or "more partial" than $t_{2}$, or, equivalently, the set of terms represented by $t_{2}$ is included in that represented by $t_{1}$.

It is also natural to think at different sets of partial terms as representations of different subsets of $\left(T_{\Sigma}\right)_{s}$.

For instance, $\left\{0, s\left(s\left(\perp_{N a t}\right)\right)\right\}$ will represent $\{0, s(s(0)), s(s(s(0))), \ldots\}$. On the other hand, a given subset of $\left(T_{\Sigma}\right)_{s}$ could be represented in many different ways. For instance, the following are alternative representations for $\left(T_{\Sigma}\right)_{N a t}$ itself: $\left\{\perp_{N a t}\right\}$, $\left\{0, s\left(\perp_{\text {Nat }}\right)\right\}$. It is worth noting that the second set differs from the first one only because it keeps the representation of 0 separated from the one of all the other values. A much more refined representation would be $\left\{0, s(0), s\left(s\left(\perp_{N a t}\right)\right)\right\}$ where a special status is granted to $s(0)$ too. Finally, notice that in $\left\{0, s\left(\perp_{N a t}\right), s\left(s\left(\perp_{N a t}\right)\right)\right\}$ there is a kind of redundancy since $s\left(\perp_{N a t}\right) \preceq s\left(s\left(\perp_{N a t}\right)\right)$.

The following definition allows to characterize all finite representations of $\left(T_{\Sigma}\right)_{s}$ made up out of partial terms which do not suffer of the above unpleasant redundancy and which differ only in the set of terms which have an explicit representation, i.e. in their "grain".

Definition 7 Cuts. Given a partial order $(D, \preceq), C \subseteq D$ is a cut of $D$ iff both the following conditions hold:

1. $\forall x, y \in C x \nprec y \wedge y \nprec x$

2. $\forall x \in D \exists y \in C$ such that $x \preceq y \vee y \preceq x$

The following defiritions will make it possible to automatically generate a finite representation $A B_{s}$ for each sort $s$ in the data type part of any given LOTOS specification. All terms textually occurring in the LOTOS specification will be explicitly represented in $A B_{\mathbf{s}}$. This is crucial since any static information on the behaviour of the GRC will in the end necessarily depend upon such terms.

Definition 8 Raw Abstract Bases: $R A B_{s}$. Let $s$ be a basic sort. The Raw $A b$ stract Base of sort $s, R A B_{s}$ is the set of all partial terms $\pi_{s}(t)$ such that $t \in$ $\left(T_{\Sigma \cup V a r}\right)_{s}$ is a term which textually occurs in the LOTOS specification. Moreover, $\perp_{s} \in R A B_{s}$ is also required. 
If $s$ is non-basic, then let $R A B_{s}^{\prime}$ be the set defined as above. Then $R A B_{s}$ will the smallest set which includes $R A B_{s}^{\prime}$ and such that all partial terms $k\left(t_{1}, \ldots, t_{n}\right)$ such that contructor $k: s_{1}, \ldots, s_{n} \rightarrow s$ occurs in $R A B_{s}^{\prime}$ and $t_{i} \in R A B_{s_{i}}$, with $R A B_{s_{i}}$ basic, belong to $R A B_{s}$ too.

In our running example we have:

$R A B_{N a t}=\left\{\perp_{N a t}, 0, s\left(\perp_{N a t}\right), s\left(s\left(\perp_{N a t}\right)\right)\right\}$

$R A B_{B o o l}=\left\{\perp_{B o o l}, t t, f f\right\}$

$R A B_{S P}=\left\{\perp_{S P}, R Q\left(\perp_{N a t}\right), R S\left(\perp_{N a t}\right), R Q(0), R S(0), R Q\left(s\left(\perp_{N a t}\right)\right), R S\left(s\left(\perp_{N a t}\right)\right)\right.$, $\left.R Q\left(s\left(s\left(\perp_{N a t}\right)\right)\right), R S\left(s\left(s\left(\perp_{N a t}\right)\right)\right)\right\}$

Definition 9 Abstract Bases: $A B_{s}$. The Abstract Base of sort $s, A B_{s}$ is the smallest cut $C$ of $\left(\Pi_{\Sigma}\right)_{s}$ such that $\forall x \in R A B_{s} \exists y \in C$ such that $x \preceq y$

For Nat, Bool, $S P$, we have:

$A B_{N a t}=\left\{0, s(0), s\left(s\left(\perp_{N a t}\right)\right)\right\}$

$A B_{\text {Bool }}=\{t t, f f\}$

$A B_{S P}=\left\{R Q(0), R S(0), R Q(s(0)), R S(s(0)), R Q\left(s\left(s\left(\perp_{N a t}\right)\right)\right), R S\left(s\left(s\left(\perp_{N a t}\right)\right)\right)\right\}$

Lemma 10. For all $s, A B_{s}$ is finite.

Definition 11 Abstract Domain : $A B S$. The Abstract Domain of sort $s, A B S_{s}$, is defined as $2\left(A B_{s}\right)$ with standard set inclusion partial ordering.

The Abstract Domain, $A B S$, for a given data type definition is defined as the $\perp$-coaleshed $s u m^{5}$ of the abstract domains for all the sorts in the type definition.

As we shall see later, intuitively the empty set, i.e. the bottom of $A B S$, denotes the undefined value, i.e. a computation which definitely fails to terminate. Any other abstract term denotes computations which may terminate and it gives information on the set of the possible values they can yield.

Abstract Functions In this section we shall show how to compute the abstract version of any function defined within an abstract data type definition $T$. To that purpose we need to define what does it mean for an abstract term to approximate a set of partial terms, which in term represents a set of possible values. We need some additional definitions:

Definition 12 Min, Max. Given a partial order $(D, \preceq)$ and $X \subseteq D$ $\operatorname{Min}(X)=\{m \mid m \in X, \nexists x \in X x \preceq m\}$ $\operatorname{Max}(X)=\{m \mid m \in X, \not \nexists x \in X m \preceq x\}$

Definition $13 \nabla$. Given a partial order $(D, \preceq)$ and $d \in D, \nabla_{d}$ is the partial order with set of elements $X=\{x \mid d \preceq x\}$ and ordering relation $\preceq \cap X \times X$.

\footnotetext{
${ }^{5}$ By $\perp$-coaleshed sum of $D_{1}, \ldots D_{n}$ we mean the coaleshed sum [12] of $D_{1}, \ldots, D_{n}$ thought as posets rather than lattices; i.e. $\perp_{1}, \ldots, \perp_{n}$ collapse into an unique element, $\perp$ in the $\perp$-coaleshed sum of $D_{1}, \ldots, D_{n}$, whereas the top elements of $D_{1}, \ldots, D_{n}$ remain distinct elements of the sum, which is no longer a lattice, but only a c.p.o.
} 
Definition 14 Approximation. Given a partial order $(D, \preceq), d \in D$, and $W \subseteq D$

1. $W$ generates $d$ iff $\exists x \in W: x \preceq d$

2. $W$ represents $d$ iff $\exists X \subseteq W: X$ is a cut for $\nabla_{d}$

3. $W$ approximates $d$ iff $W$ generates $d \vee W$ represents $d$

Definition 15 Approximation Function: $\aleph_{s}$. For all $X \subseteq\left(\Pi_{\Sigma}\right)_{s}$ :

$$
\begin{array}{ll}
\aleph_{s}(\{\}) & =\{\} \\
\aleph_{s}\left(\left\{\perp_{s}\right\}\right) & =A B_{s} \\
\aleph_{s}(\{c\}) & =\bigcup M i n\left(\left\{a \mid a \in A B S_{s}, \text { a approximates } c\right\}\right) \\
\aleph_{s}\left(\left\{k\left(p_{1}, \ldots, p_{n}\right)\right\}\right) & \text { for all constants } c \\
\aleph_{s}\left(\left\{p_{1}, \ldots, p_{n}\right\}\right) & =\aleph_{s}\left(\left\{x \mid x=k\left(x_{1}, \ldots, x_{n}\right), \bigwedge_{i=1}^{n} x_{i} \in \aleph_{s_{i}}\left(\left\{p_{i}\right\}\right)\right\}\right) \\
& \text { for constructor terms } \\
& =\bigcup_{i=1 . . n} \aleph_{s}\left(\left\{p_{i}\right\}\right)
\end{array}
$$

We shall use only finite, non-empty sets of partial terms, in which case function $\aleph$ is certainly effective y computable and total. In our running example we have for instance $\mathcal{N}\left(\left\{\perp_{N a t}\right\}\right)=\left\{0, s(0), s\left(s\left(\perp_{N a t}\right)\right)\right\}, \mathcal{N}(\{s(s(0))\})=\left\{s\left(s\left(\perp_{N a t}\right)\right)\right\}$, $\aleph(\{0, s(s(0))\})=\left\{0, s\left(s\left(\perp_{N a t}\right)\right)\right\}$.

The reason why we need the approximation function will be clear later, when we shall describe how to get the abstract version of functions from their definition. Moreover notice that when the ADT has n-ary constructors, with $n>1$, then more then one abstract term could be a "best" approximation of a set of partial terms. Consider for instance a constructor $K: S, S \rightarrow S$ for a type with a constant $a$. Assuming $A B_{S}=\left\{a, K\left(a, \perp_{S}\right), K\left(\perp_{S}, a\right), K\left(K\left(\perp_{S}, \perp_{S}\right), \perp_{S}\right), K\left(\perp_{S}, K\left(\perp_{S}, \perp_{S}\right)\right)\right\}$ we have that both $\left\{K\left(a, \perp_{S}\right)\right\}$ and $\left\{K\left(\perp_{S}, a\right)\right\}$ would be "best" approximations for $\{K(a, a)\}$. We take $\aleph(\{K(a, a)\})=\left\{K\left(a, \perp_{S}\right), K\left(\perp_{S}, a\right)\right\}$. Such an approximation is not as "good" as any of $\left\{K\left(a, \perp_{S}\right)\right\}$ and $\left\{K\left(\perp_{S}, a\right)\right\}$ but it has the advantage of making $\aleph$ be a function rather than a relation. In fact , a possible alternative could be to let $\aleph$ return the set of best approximations and then select one on the basis of some a priori criterion (i.e. lexicographic ordering). This would make safety harder to proof and it is left for further study.

Lemma 16. For all $X, Y \subseteq\left(\Pi_{\Sigma}\right)_{a} \aleph(X \cup Y)=\aleph(X) \cup \aleph(Y)$

Lemma 17. For all $X, Y \subseteq\left(\Pi_{\Sigma}\right)_{s}$ if $X \subseteq Y$ then $\aleph(X) \subseteq N(Y)$

Function Abstraction Procedure In order to compute the abstract version of all the functions defined in $T$, we generate a new system of equations starting from those in $T$ and let such abstract functions be its minimal solution.

For the sake of simplicity, in the following we shall deal with unary functions only. All definitions and results can easely be extended to $\mathrm{n}$-ary functions.

For each function $f: s^{\prime} \rightarrow s$, the set of equations defining its abstract version $f^{\mathcal{A}}$ is composed by two parts. The first one does not depend on the equations which define $f$, and it is the following one, where $p_{i} \in A B_{a^{\prime}}$ : 
1. $f^{\mathcal{A}}(\{\}) \quad=\{\}$

2. $f^{\mathcal{A}}\left(\left\{p_{1}, \ldots, p_{n}\right\}\right)=\bigcup_{i=1 \ldots n} f^{\mathcal{A}}\left(\left\{p_{i}\right\}\right)$

In order to complete the definition of $f^{\mathcal{A}}$ we have to introduce the equations for $f^{\mathcal{A}}$ when applied to singletons. The right hand side of $f^{\mathcal{A}}(\{p\})$ is obtained by selecting the equation of $f$ 's definition the left-hand-side of which matches with the partial term $p$, if any. Pattern matching is easely extended to partial terms simply considering $\perp$ as an additional constant. If no equation can be selected then the equation for $f^{\mathcal{A}}(\{p\})$ is the following:

3. $f^{\mathcal{A}}(\{p\})=A B_{s}$

Otherwise it will be the following one:

4. $f^{\mathcal{A}}(\{p\})=\mathcal{A S} \llbracket r h s(p) \rrbracket$

where $r h s(p)$ is the right-hand-side of the unique equation for $f$ which has been selected, properly instantiated according to the bindings induced by pattern-matching Function $\mathcal{A S}$ is defined as follows:

4.1) AS $\llbracket c \rrbracket \quad=\aleph(\{c\})$, for all constants $c: \rightarrow s$, or $c=\perp$

4.2) $\left.\mathcal{A} \mathcal{S} \llbracket k\left(e_{1}, \ldots, e_{n}\right)\right]=\aleph\left(\left\{x \mid x=k\left(x_{1}, \ldots, x_{n}\right), \bigwedge_{i=1}^{n} x_{i} \in \mathcal{A} \mathcal{S} \llbracket e_{i} \rrbracket\right\}\right)$, for all constructor terms

4.3) $\mathcal{A S} \llbracket f(e) \rrbracket \quad=f^{\mathcal{A}}(\mathcal{A} \mathcal{S} \llbracket e \rrbracket)$, for all functor terms.

Notice that $f^{\mathcal{A}}(\mathcal{A S} \llbracket e \rrbracket)$ is to be intended as a syntactical function application, i.e. functor term, here.

The relevant equations obtained for function $m 2$ follow:

$m 2^{\mathcal{A}}(\{0\}) \quad=\{0\}$

$m 2^{\mathcal{A}}(\{s(0)\})=\{s(0)\}$

$m 2^{\mathcal{A}}\left(\left\{s\left(s\left(\perp_{N a t}\right)\right)\right\}\right)=m 2^{\mathcal{A}}\left(\left\{0, s(0), s\left(s\left(\perp_{N a t}\right)\right)\right\}\right)$

Proposition 18. Existence of solution

Proof. The left-hand-side of the equations for the abstract functions is made up of constants (rules 1 and 3), constant functions (rules 4.1 and 4.2) and continuous operators like function application and set union (rules 2 and 4.3). Thus the existence of the least-fixed-point for the functional associated to the set of equations is guaranteed.

Finally, the abstract version of the GRC is obtained by substituting in its definition all the user-defined functions and predicates with their abstract version and the if then else operator with the following, abstract, one:

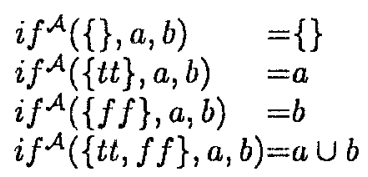


Remark. It is worth noting that, due to finiteness of all the objects involved in the computation of the abstract version of the GRC the former can be performed in finite time.

In the following table the abstract versions $i s .0^{\mathcal{A}}$ and $m 2^{\mathcal{A}}$ of functions is 0 and $m 2$ of our sample specification are given. In the third column the values for $g^{\mathcal{A}}$ are given, where $g$ is defined by $g(x)=s(m 2(x))$. Notice that those values are obtained via function $\aleph$, the use of which essential from the third row on. The keypoint here is that since the term $s(s(0))$ does not occur textually in the specification we are dealing with, it does not deserve a "distinguished" representation in $A B S_{N a t}$. On the other hand we have $g(s(0))=s(s(0))$ so we need an approximation for $s(s(0))$ in our abstract domain. Such an approximation could be $\left\{0, s(0), s\left(s\left(\perp_{\text {Nat }}\right)\right)\right\}$, which abstractly represent any natural number (i.e. is the result of $\left.\mathcal{N}\left(\left\{\perp_{N a t}\right\}\right)\right)$, but a better approximation is certainly $\left\{s\left(s\left(\perp_{N a t}\right)\right)\right\}$ which is the best indeed, given the amount of static information available.

\begin{tabular}{||c|c|c|c||}
\hline & $i s_{-} 0^{\mathcal{A}}$ & $m 2^{\mathcal{A}}$ & $g^{\mathcal{A}}$ \\
\hline\{\} & \{\} & \{\} & \{\} \\
\hline$\{0\}$ & $\{t t\}$ & $\{0\}$ & $\{s(0)\}$ \\
\hline$\{s(0)\}$ & $\{f f\}$ & $\{s(0)\}$ & $\left\{s\left(s\left(\perp_{N_{a t}}\right)\right)\right\}$ \\
\hline$\left\{s\left(s\left(\perp_{N a t}\right)\right)\right\}$ & $\{f f\}$ & $\{0, s(0)\}$ & $\left\{s(0), s\left(s\left(\perp_{N a t}\right)\right)\right\}$ \\
\hline$\{0, s(0)\}$ & $\{t t, f f\}$ & $\{0, s(0)\}$ & $\left\{s(0), s\left(s\left(\perp_{N a t}\right)\right)\right\}$ \\
\hline$\left\{0, s\left(s\left(\perp_{N a t}\right)\right)\right\}$ & $\{t t, f f\}$ & $\{0, s(0)\}$ & $\left\{s(0), s\left(s\left(\perp_{N a t}\right)\right)\right\}$ \\
\hline$\left\{s(0), s\left(s\left(\perp_{N a t}\right)\right)\right\}$ & $\{f f\}$ & $\{0, s(0)\}$ & $\left\{s(0), s\left(s\left(\perp_{N a t}\right)\right)\right\}$ \\
\hline$\left\{0, s(0), s\left(s\left(\perp_{N a t}\right)\right)\right\}$ & $\{t t, f f\}$ & $\{0, s(0)\}$ & $\left\{s(0), s\left(s\left(\perp_{N a t}\right)\right)\right\}$ \\
\hline
\end{tabular}

It is interesting to point out the kind of don't know information provided by the abstract version of predicates. Such information is represented by the abstract term $\{t t, f f\}$. So, for instance, $i s_{-} 0^{\mathcal{A}}\left(\left\{0, s(0), s\left(s\left(\perp_{N a t}\right)\right)\right\}\right)=\{t t, f f\}$ means that no information can be statically inferred on the behaviour of the test on 0 when no information is available on the argument which the test is applied to, except that it is a natural number. On the other hand, from is $0^{\mathcal{A}}\left(\left\{s(0), s\left(s\left(\perp_{N a t}\right)\right)\right\}\right)=\{f f\}$ we know that the test on 0 will yield $f f$ on any number greater then 0 . This kind of uncertainty, once generated by means of an abstract evaluation of what textually occurs in the LO'TOS specification and propagated through the abstractions of the functions used by the GRC, may give raise to uncertainty in the value of the GRC itself, so bringing to the use of choice and selection predicates in the transformation. The table below shows some interesting values of $\mathcal{G}^{\mathcal{A}}\{a\}$

\begin{tabular}{|c|c|}
\hline & $\mathcal{G}^{\mathcal{A}}\{a\}$ \\
\hline$\{R Q(0)\}$ & $\{r 0\}$ \\
\hline$\{R Q(s(0))\}$ & $\{r\}$ \\
$\left\{R Q\left(s\left(s\left(\perp_{N a t}\right)\right)\right)\right\}$ & $\{r\}$ \\
\hline$\left\{R Q(0), R Q(s(0)), R Q\left(s\left(s\left(\perp_{N a t}\right)\right)\right)\right\}$ & $\{r 0, r\}$ \\
\hline$\{R S(0), R S(s(0))\}$ & $\{s 0, s\}$ \\
\hline
\end{tabular}

From the above table it should be clear that if no information is available on $n$ in actions like $\langle a, R Q(n)>$ then all that can be said about the possible outcomes of 
the GRC on those values is that they are $r 0$ and $r$. Some uncertainty is still present but this is better then saying that the set of possible outcomes is $\{b, r 0, r, s 0, s\}$ which would be the case when no information at all is available on the values passing through $a$, except their type (namely $\mathcal{G}^{\mathcal{A}}\{a\} A B_{S P}$, not shown in the table).

Finally, it is worth noting that $m 2^{\mathcal{A}}\left(\left\{0, s(0), s\left(s\left(\perp_{N a t}\right)\right)\right\}\right)=\{0, s(0)\}$ essentially tells us which is the co-domain of $m 2$. When such information for any function $f: s 1 \rightarrow s 2$ cannot be computed statically, one will get $f\left(A B_{s 1}\right)=A B_{s 2}$.

The last step before defining the transformation is the definition of a function, $\mathcal{V}$, which maps LOTOS value expressions into their abstract values. It takes a veriable identificr/abstract-value binding function (environment) as well.

Definition 19 Abstract Data Evaluation. $\mathcal{V}_{s}: V e x_{s} \rightarrow A E n v \rightarrow A B S_{s}$ where: $A E n v=$ VIdentifiers $\rightarrow A B S_{s}$

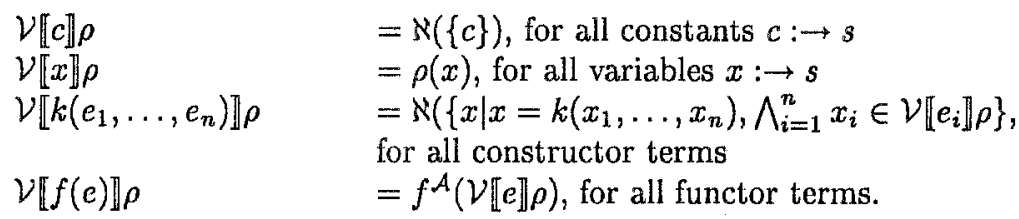

Notice that $f^{\mathcal{A}}(\mathcal{V} \llbracket e \rrbracket \rho)$ denotes the result of the application of the actual function $f^{\mathcal{A}}$, as defined by the appropriate system of equations, to $\mathcal{V} \llbracket e \rrbracket \rho$.

Correctness of the Abstract Interpretation In this section we shall prove that the abstract interpretation obtained according to the technique defined in the previous sections is safe, that is it provides correct information. We shall proceed according to the approach known as logical relation [1]; other approaches $[4,5,6,7]$ use Galois insertions as safety criterion. We shall first define the correctness requirement. Then we shall prove that the abstraction function $\aleph$ meets such requirement. Finally we shall prove the main proposition which states that correctness is preserved by abstract functions. It is worth pointing out that correctness is proved w.r.t. a fixpoint semantics of LOTOS abstract data types, which is not the standard one. The relation between the two semantics is not investigated here. Anyway the data types definition part of LOTOS is likely to be changed into a functional-like one [19].

We start by extending our concrete semantic sets with the undefined elements and by defining the proper partial orders.

Definition 20 Concrete semantic domains. For all sorts $s$ $\omega_{s} \in\left(T_{\Sigma_{c}}\right)_{s}$ and $\omega_{s} \in\left(\Pi_{\Sigma}\right)_{s}$

For all $p_{1}, p_{2} \in\left(\Pi_{\Sigma}\right)_{s} p_{1} \sqsubseteq p_{2}$ iff $p_{1}=\omega$ or $p_{1}=p_{2}$

We shall assume natural extension for all functions, i.e. for all $f: s \rightarrow s^{\prime} f\left(\omega_{s}\right)=$ $\omega_{s^{\prime}}$. Moreover, for all (finite) sets $P \subseteq\left(I_{\Sigma}\right)_{s}$ we define $\aleph(P \cup\{\omega\})=\aleph(P)$. Thus $\aleph(\{\omega\})=\{\}$. 
Definition 21 Concretization Function: $\gamma_{s}$.

$\begin{array}{ll}\gamma_{s}(\{\}) & =\left\{\omega_{s}\right\} \\ \gamma_{s}\left(\left\{p_{1}, \ldots, p_{n}\right\}\right) & =\bigcup_{i=1, \ldots n} \gamma_{s}\left(\left\{p_{i}\right\}\right) \\ \gamma_{s}(\{p\}) & =\operatorname{Max}\left(\nabla_{p}\right)\end{array}$

Lemma 22. For all $a_{1}, a_{2} \in A B S_{s} \gamma\left(a_{1} \cup a_{2}\right)=\gamma\left(a_{1}\right) \cup \gamma\left(a_{2}\right)$

Lemma 23. For all $a_{1}, a_{2} \in A B S_{s}$ if $a_{1} \subseteq a_{2}$ then $\gamma\left(a_{1}\right) \subseteq \gamma\left(a_{2}\right)$

Lemma 24. $\left(T_{\Sigma_{c}}\right)_{s} \subseteq \gamma\left(A B_{s}\right)$

It is interesting to point out that $\omega \in \gamma(a)$ for any abstract term $a$. So the empty set abstractly represents all those computations the result of which is undefined (i.e. those computations which definitely fail to terminate) whereas any other abstract term $a$ represents computations the result of which may be not undefined (i.e. they may terminate) in which case it also gives some information on the possible output values.

Definition 25 Correctness relation: $\mathcal{C}$ (read "correctly represents"). For all $a \in A B S_{s}, t \in\left(T_{\Sigma_{c}}\right)_{s} a C t$ iff $t \in \gamma(a)$

So, essentially, $a$ correctly represents $t$ if and only if $t$ can be generated from $a$ via instantiation of some partial term in $a$

Lemma 26. For all $p \in\left(\Pi_{\Sigma}\right)_{s}, t \in\left(T_{\Sigma_{c}}\right)_{s}$, if $p \preceq t$ then $t \in \gamma(\aleph(\{p\}))$

Corollary 27. For ail $t \in\left(T_{\Sigma_{c}}\right)_{s} \aleph(\{t\}) C t$, i.e. the abstraction function is correct.

Lemma 28. If $a_{i} \mathcal{C} t_{i}$ for $i=1 \ldots n$ then $\aleph\left(\left\{k\left(x_{1}, \ldots, x_{n}\right) \mid x_{i} \in a_{i}\right\}\right) \mathcal{C} k\left(\dot{t}_{1}, \ldots, t_{n}\right)$

Proposition 29 Safety of the abstract interpretation. For all $a \in A B S_{s}, t \in$ $\left(T_{\Sigma_{c}}\right)_{s} f: s \rightarrow s^{\prime}$ with $f^{\mathcal{A}}: A B S_{s} \rightarrow A B S_{s^{\prime}}$ defined according to the abstraction procedure, the following holds: $a \mathcal{C} t \Rightarrow f^{\mathcal{A}}(a) \mathcal{C} f(t)$

Proof. We make use both of computational and structural induction. Letting $g_{i}$ denote the $i$-th approximation of the minimal fixpoint for the equations for function $g$, it has to be proved that for all $i$ it holds $a \mathcal{C} t \Rightarrow f_{i}^{\mathcal{A}}(a) \mathcal{C} f_{i}(t)$. The (computational) induction step requires induction on the structure of $a$ in turn. Finally it must be proved that if the proposition holds for all approximations of the minimal fixpoint then it holds also for the fixpoint itself. The details of the proof are given in [9].

\subsection{Definition of the Transformation}

The transformation is defined as a function $\mathcal{T B}: B e x \rightarrow A c \rightarrow A E n v \rightarrow B e x$. In [9] the function is formally defined by structural induction on the set of all LOTOS behaviour expressions (:Bex) and takes the abstract version of a GRC $(: A c)$ and an 
abstract environment (:AEnv) as extra arguments. It obviously returns a behaviour expression as result. $\mathcal{T B}$ acts mainly on action prefix, propagating throughout the whole specification the changes which it makes and also performing some simplifications, on the basis of static "data-flow" information recorded in the abstract environment. In the sequel she shall discuss the behaviour of $\mathcal{T B}$ only on "input" action prefix $g ! e ; B$ and in an informal way.

Let $\mathcal{R}$ be a GRC. Let also $\rho$ the abstract environment collecting all the static information on the possible values which variables can be bound to in the context in which $g ! e ; B$ is to be transformed. Now, if for this particular occurrence of $g$, it is statically decidable that the GRC gives only one value $g^{\prime}$ as result, i.e. $\mathcal{R}^{\mathcal{A}}\{g\}(\mathcal{V} \llbracket e \rrbracket \rho)=\left\{g^{\prime}\right\}$, then $\mathcal{T} \mathbb{B} \llbracket g ! e ; B \rrbracket \mathcal{R}^{\mathcal{A}} \rho=g^{\prime} ! e ; \mathcal{T} \mathcal{B} \llbracket B \rrbracket \mathcal{R}^{\mathcal{A}} \rho$. If, on the other hand, $\mathcal{R}^{\mathcal{A}}\{g\}(\mathcal{V} \llbracket e \rrbracket \rho)=\left\{g_{1}, g_{2}, \ldots, g_{k}\right\}$, then $\mathcal{T} \mathcal{B} \llbracket g ! e ; B \rrbracket \mathcal{R}^{\mathcal{A}} \rho=$ $g_{1} ! e\left[p_{1}(e)\right] ; \mathcal{T} \mathbb{B} \llbracket B \rrbracket \mathcal{R}^{\mathcal{A} \rho}$ [] $g_{2} ! e\left[p_{2}(e)\right] ; \mathcal{T} \mathcal{B} \llbracket B \rrbracket \mathcal{R}^{\mathcal{A}} \rho$ [] $g_{k} ! e\left[p_{k}(e)\right] ; \mathcal{T} \mathbb{B} \llbracket B \rrbracket \mathcal{R}^{\mathcal{A}} \rho$ where $p_{j}$ is the unique predicate such that $p_{j}(v) \Rightarrow \mathcal{R} g v=g_{j}{ }^{6}$.

In conclusion, given a process definition whose behaviour expression is $B$, with type definitions $T$ (with sorts $s_{1}, \ldots, s_{k}$ ) and local process definitions $P_{1}, \ldots, P_{n}$, and a GRC $\mathcal{R}$ the transformed process definition is given by $\mathcal{T} \mathbb{B} \| B \rrbracket \mathcal{R}^{\mathcal{A}} \Omega$ where $\Omega x=A B_{s_{i}}$ for all variable identifiers $x$ of sort $s_{i}$. Obviously the transformation has to be applied to all $P_{i}$ in turn.

Proposition 30 Correctness of the transformation. For any behaviour expression $B$ and $G R C \mathcal{R}, B \sim_{\Phi} \mathcal{T B} \llbracket B \rrbracket \mathcal{R}^{\mathcal{A}} \Omega$ provided that for all $g 1, g 2 \in$ Gates, $v 1, v 2 \in$ Value: $\mathcal{R} g 1 v 1=\mathcal{R} g 2 v 2 \Rightarrow(v 1=v 2 \Rightarrow g 1=g 2)$

Proof. Under the assumption that the above requirement is met, it is trivial to prove that the function given below is a bijection from the set of actions of $P$ to that of $Q$ : $\phi(x<v>)=(\mathcal{R} x v)<v>$ with $x \notin\{i, \delta\}^{7}$

$\phi(i)=i$

$\phi(\delta<v>)=\delta<v>$

The second step is to show that for all behaviour expressions $B$, the pair $<B, \mathcal{T B} \llbracket B \rrbracket \mathcal{R}^{\mathcal{A}} \Omega>$ is in some $\phi$-bisimulation relation, which can be proved by structural induction on $B$, using the definition of $\mathcal{T B}$. In [10] the proof is given in detail, under the simplifying assumption that $\mathcal{R}$ does not depend on the actual value of its second argument but only on its sort. The extension to the more general case does not introduce any conceptual complication.

\footnotetext{
${ }^{6} p_{j}$ can easely be derived from the definition of $\mathcal{R}$. Notice that such a predicate may contain a test on the sort of $v$, which must obviously be removed in the selection predicate.

${ }^{7} i$ and $\delta$ denote LOTOS internal, respectively, successful termination actions.
} 


\section{Further Work}

Further work is required in order to improve our technique. For instance, since the transformation is very much sensitive to the particular way in which the GRC is defined, reasonable guidelines or even further syntactical/static semantics restrictions on the GRC meta-notation are needed in order to get real advantage of available static information.

Moreover, the transformation itself can be improved in many ways. Better simplifications can be achieved by means of using abstract interpretation of boolean expressions in selection predicates and guards and then using the abstract values for properly modify the environment in order to keep track of the abstract data flow.

An analysis which keeps track of the abstract values of the parameters present at every instantiation could make it possible to discover that the abstract value of all the actual parameters associated to a given formal parameter in all the instantiations of a process definition within a given behaviour expression are the same, so allowing forther optimizations in the associated process definition.

The current, simplified, implementation of the transformation in the LOTOS Integrated Tool Environment - LITE [21] imposes that the GRC cannot depend on the actual values exchanged throughout a given gate but only on their sorts. We are now starting developing a new version of the tool where such a requirement is removed and the abstract domain construction presented in this paper is implemented.

Finally, we are thinking at further applications of our technique. An interesting application of the analysis technique we have described in the present paper is to use it in order to study the dependence on data of a system's behaviour or of those aspects of the behaviour one is interested in. Suppose for instance we are interested in studying safety or liveness properties of a communication protocol. It is then likely that for our purposes we can just abstract away from everything in the protocol which has to do with "user-data" and just concentrate on "control-data" since they influence the behaviour of the protocol w.r.t. the properties we want to reason about. In this way we can reduce the complexity of the problem and probably move from an infinite object, i.e. the "whole" protocol, defined on user data too, towards a finite object which represents the "control part" of the protocol. Then suitable tools (usually working only on finite objects) can be used for checking. We think our technique can profitably be used for studying such dependence on data of both the LOTOS specification and the particular (modal) logic formula which expresses the desired property.

\section{Acknowledgments}

The intuitive ideas behind the technicalities described in this paper come from discussions with Elie Najm and Rom Langerak during an unforgettable ESPRITLOTOSPHERE meeting in Venice, March 1991.

\section{References}

1. S. Abramsky, C. Hankin, ed.: Abstract Interpretation of Declarative Languages. Computers and their Applications. Ellis Horwood, Chichester, U.K. 1987 
2. T. Bolognesi, E. Brinksma: Introduction to the ISO specification language LOTOS. Computer Network and ISDN Systems - Vol. 14, No 1, 1987

3. E. Brinksma (Ed.): LOTOS - A formal description technique based on the temporal ordering of observational behaviour. ISO IS8807, February 1989, ISO, Geneva

4. P. Cousot, R. Cousot: Static determination of dynamic properties of programs. Proc. of the 2nd Int. Symp. on Programming Languages. Dunod, Paris 1976

5. P. Cousot, R. Cousot: Abstract Interpretation: a unified lattice model for static analysis of programs by construction or approximation of fixpoints. Proc. of the 4th ACM Symp. on Principles of Programming Languages

6. P. Cousot, R.Cousot: Static determination of dynamic properties of recursive procedures. Proc. of IFIP Conf. on Formal Description of Programming Concepts. NorthHolland 1977

7. P. Cousot, R.Cousot: Abstract Interpretation Frameworks. Journal of Logic and Computation, 1992 (to appear)

8. H. B. Curry, R. Feys: Combinatory Logic. Vol. I. North Hollnad, 1968

9. F. Giannotti, D. Latella: Gate Splitting in LOTOS Specifications By Using Abstract Interpretation. CNUCE Internal Report C92-13

10. D. Latella: Correctness of the IPR transformation. ESPRIT Project 2304 - LotoSphere, ref. Lo/WP1/T1.2/CNUCE/N0021- 1991

11. R. Milner: A Calculus of Communicating Systems. Lecture Notes in Computer Science vol. 92, Springer-Verlag, 1980

12. J. Stoy: Denotational Semantics: the Scott-Strachey Approach to programming languages Theory. The MIT Press 1979

13. J. Schot, L. Pires (eds.): Final deliverable of the PANGLOSS Architectural Task Results of the ESPRIT/PANGLOSS project - ref. P/AT/137, part II, section 4.3.4.1. University of Twente, Enschede, Netherlands, December 1989

14. L. Pires (Ed.): The LotoSphere Design Methodology - I: Basic Concepts. ESPRIT Project 2304 - LotoSpliere, Task 1.1 Deliverable, ref. Lo/WP1/T1.1/N0045/V03 - 1992

15. The LotoSphere Design Methodology - I: Guidelines. ESPRIT Project 2304 - LotoSphere, Task 1.1 Deliverable, ref. Lo/WP1/T1.1/N0044/V03 - 1992

16. The LotoSphere Design Methodology - I: Illustrations. ESPRIT Project 2304 - LotoSphere, Task 1.1 Deliverable, ref. Lo/WP1/T1.1/N0046/V03 - 1992

17. G. Yadan: (Ed.) The LotoSphere Design Methodology - I: Experience in industrial environment. ESPRIT Project 2304 - LotoSphere, Task 1.1 Deliverable, ref. Lo/WP1/T1.1/N0047/V03 - 1992

18. T. Bolognesi (Ed.): Catalogue of LOTOS Correctness Preserving Transformations ESPRIT Project 2304 - LotoSphere, Task 1.2 Deliverable, ref. Lo/WP1/T1.2/N0045/V03 $-1992$

19. LOTOS Enhancements. ESPRIT Project 2304 - LotoSphere, Task 1.4 Final Deliverable $-1992$

20. P.H.J. van Eijk, C. A. Vissers, M. Diaz (Ed.): The Formal Description Technique LOTOS. North-Holland

21. P.H.J. van Eijk: Tool demonstration: The LotoSphere Integrated Tool Environment Lite. Proc. FORTE'91, North-Holland, 1992

22. K. Warkentyne: The Application of Transformations in T3.2 (MiniMail+ISDN). ESPRIT Project 2304 - LotoSphere, ref. WP1/T11/ASCOM/N0015, Feb. 1992 